\title{
Phobia Treatment with Face-Your-Fear VR App
}

\author{
Samia Loucif', Joao Negreiros¹, Batoul Haj Stifi'², Rahma Omar Abdel Rahman², Sara Iqbal² \\ ${ }^{1}$ College of Technological innovation, Zayed University, Abu Dhabi, UAE \\ ${ }^{2}$ Software Engineering Department, ALHOSN University, Abu Dhabi, UAE \\ Email: samia.loucif@zu.ac.ae Joao.negreiros@zu.ac.ae, Batoul.h.stifi@gmail.com,rahoom.elrawy@gmail.com, \\ contact@programmerszone.ae
}

How to cite this paper: Loucif, S., Negreiros, J., Stifi, B.H., Rahman, R.O.A. and Iqbal, S. (2021) Phobia Treatment with Face-YourFear VR App. Journal of Computer and Communications, 9, 27-43.

https://doi.org/10.4236/jcc.2021.910003

Received: September 3, 2021

Accepted: October 8, 2021

Published: October 11, 2021

Copyright ( 2021 by author(s) and Scientific Research Publishing Inc. This work is licensed under the Creative Commons Attribution International License (CC BY 4.0).

http://creativecommons.org/licenses/by/4.0/ (c) (i) Open Access

\begin{abstract}
Technology plays an important role in people's daily life, for they depend on it to perform many tasks. Among the prominent technologies are smartphones and Virtual Reality (VR). Developing mobile apps and integrating VR technology allow users to experience an artificial environment as if it is real such as the simulation of phobias. This research paper proposes a mobile application named Face-Your-Fear (FyF) integrating VR to help any user to live a cybernetic experience, overcome the illness, know about phobia symptoms, and get extra information on two types of phobia: aquaphobia and achluophobia. Its architecture, features, development, interfaces and limitations are presented, including the main results of the qualitative survey to users.
\end{abstract}

\section{Keywords}

Phobia, Technology, Virtual Reality, Android App, Face-Your-Fear (FyF)

\section{Introduction}

Phobia comes from the Greek word Phobos and it is defined as an "exaggerated usually inexplicable and illogical huge fear of a particular object" [1] like cats, insects or crowded places. It is a disease that can affect people's daily life and, in some cases, it can lead to depression. Phobia is a component of anxiety mental illnesses that causes intense fear of specific objects, situations or some types of activities [2]. Having phobia is much more serious than a normal logical fear, that is, a specific part of the brain is found to be responsible of some kinds of phobias [3]. Phobia is categorized as specific (simple), social or agoraphobia (complex). Social phobia regards the fear of participating in certain activities in public like eating or speaking. These people tend not to attend any event like weddings or parties, which affects their social lives and with a greater chance to get depression due to their isolated lifestyle. 
Phobias can be caused by two main factors, either genetically or past experiences. Phobia symptom is mostly about getting a panic attack, which can be described in terms of racing heartrate, shortness of breath, fast/rapid speech or inability to speak, dry mouth, nausea, increased blood pressure, shaking, chest pain, choking sensation (in some cases), dizziness and sweating [4].

Often, phobic people ignore their symptoms and do not get psychotherapy due either to the social perspective about visiting psychologists or cannot afford the cost of psychotherapy. Two passible types of treatments exist. One is via medication, where the patient will take both anti-depressants and anti-anxiety pills that help them to calm both physical and emotional reaction to their fear. The second one is Cognitive Behavioral Therapy (CBT), where the patient is exposed to the phobia itself. This exposure is accomplished in continuous sessions, setup by the therapist as levels and he must be monitoring the patient while applying the treatment [5].

In this latter process, the patient has to deal with the phobia by being exposed to an environment that has elements that cause the phobic reaction. Some of the negative reactions are patient leaving the treatment or, in extreme cases, pass out. In addition, in some local communities, psychotherapy is not available, so even if the patient wants to be treated there is no place where to go or $\mathrm{s} / \mathrm{he}$ must travel afar to get the right treatment.

The research on VR in education is still in an early stage and to measure the impact of VR mobile apps on the learning motivation of undergraduate health science students at the University of Cape Town is proposed by [6]. According to these authors, attention, relevance, confidence and satisfaction (ARCS) model guided the understanding of the impact of VR on student motivation where differences in student learning motivation before and after using VR apps were conducted (78 participants). Their results showed that using VR apps increased the learning motivation of students, including in all ARCS dimensions.

Taking full advantage of this current technology, the present research proposes an integrated system of smartphone and VR technologies for phobia treatment. The proposed app assists patients to take the sessions at any time with ease and let them face their own fear. It is designed to provide an affordable therapy instead of costly treatments and avoid long waiting hours for appointments. Since the environment is be controlled and graded, they can repeat the sessions at their own will. As expected, there are several levels of treatment that the patient might be gradually exposed to. These levels will be available and, as the patient becomes more comfortable, s/he can move to a more intense level. Furthermore, patients are provided with several types of phobias and respective symptoms. The app assists the user to search the psychiatrist in the selected city (UAE in our case), as well.

This paper is organized as follows besides the present introduction. Section 2 discusses related work while the following one presents the proposed Face-YourFear (FyF) App concerning its architecture and features. Finally, Section 4 and 5 concludes this paper but not forgetting a user study to verify the present app ef- 
fectiveness.

\section{Related Work}

VR is a computer-generated simulation where people experience the present. It has been developed with the aim to allow users to handle data with ease by offering an active and dynamic way to perceive and experience fake data. VR is quite intuitive, interactive and it is quick in replying to the user's requests [7]. It has gained a great interest in its application in several fields, including automotive industry, entertainment, healthcare, architecture, education and military training.

The extent of immersion identifies how much a user is involved with the environment. It can also be regarded as how powerful the user attention is focused on the current task or situation. Immersion presence is commonly based on several parameters such as interaction level, image quality, stereoscopic view and the rate update of the display. Theoretically, VR can be classified as non-immersive systems with small implementation of VR techniques, semi-immersive systems with high quality graphics in association with big displays and fully-immersive systems which provide the most effective and real virtual experience to users but requiring the usage of head-coupled display [8].

As stated earlier, VR has been applied to several fields, embracing medicine to treat phobic patients. It gives the reality feeling so the patient may experience the object or situation to overcome their fear. More importantly, if both visual and sounds are applied to the environment, this enhances and increase the VR immersion, that is, sounds or music can grab user's attention and lower their anxiety, strong emotions and feelings [9] [10] [11] [12].

Smartphones are becoming an integral part of our lives. They possess high computing capabilities, equipped with a variety of sensors, Internet connectivity, high resolution and touch screen, which led to the development of tremendous of applications in several areas. Recently, VR glasses have been designed for smartphones, where its display is viewed as VR content.

Social and environmental purposes are also key areas in VR and augmented reality (AR). PeakLensVR is a VR mobile app that enables users to capture panoramic mountain images with their mobile and later visualize such images [13]. This application exploits a multi-stage data processing pipeline, which comprises the following steps: 1) the acquisition of a sequence of frames with the mobile phone camera and their annotation with sensor readings captured during the shooting session; 2) the creation of a panoramic image from the acquired frames, with state-of-the art stitching algorithms; 3) the registration of the panoramic image to the mountain skyline in view, by comparing the image skyline with a virtual profile extracted from the NASA SRTM Digital Elevation Model of the Earth; 4) the enrichment of the registered panoramic image with markers and metadata (name, altitude, etc.) of the peaks in view, by querying the OpenStreetMap GIS. 
Museums offer technological and digital options to enrich the user experience in a visit. However, questions arise like which exhibition/museum could I visit? How to tour it and get the best experience? A hybrid approach to make recommendations for museum visits is proposed by [14], including Internet of Things architecture of beacons, data mining and machine learning. The result is a customized tour with augmented reality that contains a set of recommendations of how to visit a set of museums and obtain a better experience of the visit whose prototype is available in Google Play and named "Historic Center".

The first study assessing the effectiveness of VR therapy was published in 1995 by Rothbaum et al. [15]. Another study showed that VR facilitated people suffering from acrophobia [16]. Virtually Better [17], a company established in 1996, development a VR software and equipment for the psychologists and clinics whose main features include VR environments for airplanes, elevators and thunderstorm for phobic patients. This treatment is offered in setting up with a normal office chair. There is a headgear (which is like an oversized cycle helmet with earpieces) and a flip-down display, connected to patient's desktop computer. Then, the user may start the session and experience the environment in addition to the feature of head movements. However, the whole system has some drawbacks such as the use of heavy equipment and being quite expensive; it could only be afforded by the therapists for long term use. It was mainly used for flying phobia early treatment.

Previsl [18] is a Spanish company that develops and sells VR software. Similar to Virtually Better, it offers VR Environments for airplanes, elevators (closed spaces and height phobia) and thunderstorms in 13 countries. It provides preparatory flight travel VR scenes such as packing of traveling bags, sitting in terminal and announcements of flights to make the person more comfortable with the environment and anticipate and prepare him/her for the upcoming event. It offers a computer-based VR therapy which is expensive.

CleVR [19] was established in 2010 in the Netherlands with the aim to offer VR environments for various domains. They provide software for VR Exposure Therapy (VRET) in association with Delft University of Technology. The software is being used to treat flying and social phobia. The patients can experience the virtual environment with the help of HDM and observe the entire environment all around them. Meanwhile, therapists have full control on changing the environment by increasing or decreasing the complexity level.

Spider-World [20] is a computer-based VR therapy with an additional ability feature to feel the object. As the name suggests, it is used to treat patients with spider phobia. Under this treatment, the patient can touch a virtual spider with the help of a cyber hand, generating the illusion of a spider touch. Certainly, this type of tactile augmentation doubled the effectiveness of VR treatment by improving the patient confidence level towards the phobic stimulus.

Phobos is a VR software developed by PsyTech LLC [21]. It was specially designed to treat phobic patient with a graded approach. The software is based on 
VR Exposure Therapy (VRET). It provides private environments for patient to manage their anxiety symptoms, including interactive VR environments of some phobias such as flying, height, spiders and crowded spaces.

VR medical center [22] was established in the USA with the purpose to treat phobic patients with the most suitable, affordable and convenient way. The medical center is based on VR equipment and software required, making the treatment possible. The center currently treats several phobias, including flying, fear of driving, public speaking and thunderstorm phobia. The medical center uses the CAVE and HDM setups to treat patients. It was a winner of patients Choice Awards in 2015.

In addition, numerous mobile applications have been developed under this context. Beat Social Phobia [23] was developed by Andrew Johnson, a Clinical Hypnotherapist, and released by HiveBrain Software in 2013. It is an app that provides audio instructions from the therapist to think in a more positive way. It helps to identify the specific phobia, its symptoms and how to deal with it. It helps to become more confident, relaxed and stay at calm in social places. The Beat Social Phobia app session are twenty-six minutes long and divided into four parts: introduction, relaxation, social phobia and awakening. However, it does not provide any video tutorials or immersive environments for the phobic patient. Thus, the patient cannot experience the real (virtual) situation.

Byten Phobia Treatment [24] was developed by Paul Mckenna in 2013 and released by Once Byten Limited. It runs on iOS and provides audio session. The patient can select one of the phobias from the list, listen to the sessions at their own pace and try to think in a different manner towards the phobic agent. This app has some weaknesses such as it does not offer videos or exposure therapy environment for agents which do not help the real experience of the phobia itself. The option to reverse the audio session to a specific point is not offered, so the user must listen to the whole session again. Quite often, the app gets irresponsive and does not response at all.

Cure Phobias and Overcome Fear [25] is an Android app developed in 2009. It is offered by MasterMind Apps and offers audio session of therapy by instill a positive thinking in users to overcome their personal problems. It provides a textual description about phobia and provides tips to overcome them. It contains some flaws such as easy lacking of user interface and the audio session is quite long, causing loss of attention. Sometimes, audio does not play at all and, occasionally, becomes unresponsive.

Arachnophobia Free app [26] has been developed in association of two leading psychiatrists to treat people with spider phobia and offered by Thrive Technologies Limited. The app is supported by iOS and it is based on VRET where patients are exposed to spiders in a graded manner. In early stages, the spider is represented as a little cute pink character named "Itsy" and, as the game progresses, it turns to a real looking spider. The user can retake the session to get over their fear. The app provides guides by the help a virtual doctor called Dr. Freeman. 
This app [27] is of high quality and has been winner of CUTEC at Cambridge University. However, its drawback concerns treatments a single type of phobia only.

Spider Fobia Cardboard [28] was developed by Alterego Games and is a VR app to facilitate people suffering from Arachnophobia by exposing them to environments full types of spiders. However, this app holds a limited number of scenes, the animation is quite slow and the transitions between the scenes of the phobia environment (as the user makes movement) are not smooth.

Created by Malmum Developers, VR Height Phobia aims height phobia [29]. It has three levels where the users may easily navigate through them and may repeat any level until they become comfortable. However, it has poor graphics and the user has to launch the app again after completing each level.

Developed by Virtual Speech, Public Speaking VR [30] application goals are public speaking phobia treatment. The scenes are provided with lot of people seated and some distractions are created with noise and voice of people talking. This helps the app user to boost him/her self-assurance to face the real settings confidently. Some of the setbacks includes blurred transitions while, sometimes, the splitting screen does not work.

\section{The Proposed App}

\subsection{Research Methodology}

To develop a fully functional and good quality VR mobile app that helps phobic treatment, a survey was conducted to people with different age, gender, professions and some doctors. The data collected provided a quantitative insight into phobia related questions. As well, the obtained feedback from Survey Monkey helped in the elicitation of requirements and features to be consider when developing an app.

The gender participation ratio was $64 \%$ females and $36 \%$ males. The age distribution was as follow: $70 \%$ in the range $18-24,13.5 \%$ in the range $25-34$, $10 \%$ in the range $35-44$ and $6.5 \%$ in the range $45-54$. Of the 77 respondents, $72.73 \%$ live in the UAE while the remaining respondents live outside the UAE (Palestine, Syria, Turkey, KSA, Germany, Egypt). The main purpose of knowing the respondent's location is closely related to some countries phobias patterns. This happens because phobias are frequently based on each local environment factor.

Participants were given a phobia list to select the one(s) they suffer. The results were as follows: $4.22 \%$, arachnophobia; $8.42 \%$, claustrophobia; $6.85 \%$, glossophobia; $13.51 \%$, aquaphobia; $11.81 \%$, acrophobia; $12.43 \%$, achluophobia; $6.85 \%$, avidophobia; $8.56 \%$, entomophobia; $4.43 \%$, hemophobia; $9.59 \%$, ailurophobia; $13.33 \%$, other options such as war, fighting, sexual assault, my wife, snakes, cockroaches or not having phobia at all.

Another inquiry was closed related to whether the participants (who suffer from a type of phobia) are link to a specific event. $84.42 \%$ of them simply stated 
no. Furthermore, to help understand the symptoms that occur when a person encounters the phobic agent, the most common affect (50\%) is rapid heartbeat. Some respondents specified other side-effects such as trouble breathing, panic attacks, shaking, nausea, run away and sweating.

For those who have phobia, $54.67 \%$ of the respondents affirmed that they searched already for a specialist. Concerning the preference of way of treatment, $17.14 \%$ favors treatment with pills while $82.86 \%$ prefer treatment by exposure to the feared object/situation. The last question was related to the adoption of a mobile application for phobia treatment. $63.51 \%$ were positive towards this idea.

\subsection{Architecture}

The FyF App consists of a three-layer framework consisting of presentation, business and data layers. The former contains the user interfaces which represent the scenes and developed in Unity. The business is a service layer that contains Java (JS) and C\# scripts to interact with the data layer for processing purpose and to display the scenes and give the appropriate feedback to the end-users.

This VR-based phobia app runs on Android OS for smart phones. It also requires the use VR glasses such as Google Cardboard, which come in an affordable cost and in order to experience the phobia environment. The clients are phobic patients and the psychiatrist. The patient is the one that experiences phobia environments, searches for nearby psychiatrist and sends experience feedback to the specialist. For the psychiatrist, s/he may use the app to receive the patient experience outcome and discusses/follows up with the patient.

Face-Your-Fear targets exposure therapy to phobic patients in both safe and controlled environment. The present status of the app includes two types: aquaphobia and achluophobia, Moreover, it provides a virtual environment where patients are gradually exposed to the stimulus (or an object) and able to face their fear. A patient may select the appropriate phobia from the menu and begin his/ her VR Exposure Therapy (VRET). The user can choose the environment and view it virtually. This is a graded and continuous process that helps the patient to be treated at his/her own pace. This also ensures the privacy of the patient. $\mathrm{S} /$ he has the choice to play music to relax and to decrease anxiety. Certainly, listening to music has a great comforting influence on minds, particularly slow, quiet music to slow down the pulse and heart rate. In other words, not only visual and graphics but audio should be given same priority. In addition to the therapy sessions, the user may search for types of phobia, their symptoms and how each type of phobia can be treated. Additionally, the user can search for the nearest psychiatrist.

\subsection{Features}

Our proposed app differs from existing applications in several ways: 1) It offers more than one environment for therapy exposure, which helps in providing a wider range of phobias to be treated. Currently, it includes two types and planned 
to be extended to other ones. 2) The graphics used are visually realistic with customized audios, suitable with each environment. Certainly, enhanced computer graphics provide a realistic immersive experience to any patient. 3) The level concept with more complex environment is a new notion and provides a graded approach towards the patient's phobia. 4) The short and long session option is also an additional feature not found in many similar VR apps. It helps the patient to get familiar with environments during short session and take long ones for 30 minutes. 5) The search nearby psychiatrist feature is also not found in other analogous applications.

Some non-functional requirements were also taking into consideration such as usability, flexibility, performance, interoperability, maintainability and security since they are highly valuable in determining the effectiveness of the functions provided by the app. Quite often, they are also known as quality attributes. For instance, maintainability is the factor concerned with system restoring after the occurrence of a failure while reliability emphasizes the application performance to deliver functionality without unnecessary delays and failures. Therefore, FyF should be able to respond to users promptly allowing him/her to navigate and use the application with no errors or halts.

Performance regards the property concerned with the response time. As the mobile device will be moved and turned upside down frequently, user's view will be changed accordingly. Thus, the app should be able to update the screen quickly, accurately and without delays. In our particular case, a response time of one second or less should be the appropriate setting to provide a "believable" VR system.

\subsection{Development}

In its development, several software packages (see Figure 1 and Figure 2) have been used to create realistic and high-quality graphics, including animations and audios to provide realistic and natural experience for the end-user. The game engine choice was Unity3D with its nice feature of multiple OS platforms. In a reference note, Unity is a game engine that supports simulations, desktop, websites and apps, including an SDK for virtual and augmented reality. Google Sketch Up, a powerful and user-friendly Computer Aided Design (CAD) created realistic effects too. Max 3D, used to develop 3D animations, models and characters, provided realistic and professional graphics and it has been used to develop some extra VR environment features. Audacity audio editor provided multiple features of music editing and merging recording sounds. It was used to develop sound effects of water waves and darkness sound at night for the aquaphobia and achluophobia environments. Blender Software has been used to create 3D models and developing characters, videos and audios. It offers animations and highquality graphics. Besides, it also supports natural environment and object creation such as raindrops, flames, smoke, rainbows and sky movements. Cardboard SDK, a VR based support compatible with Unity to develop VR applications, has been also exploited to develop immersive environments. 


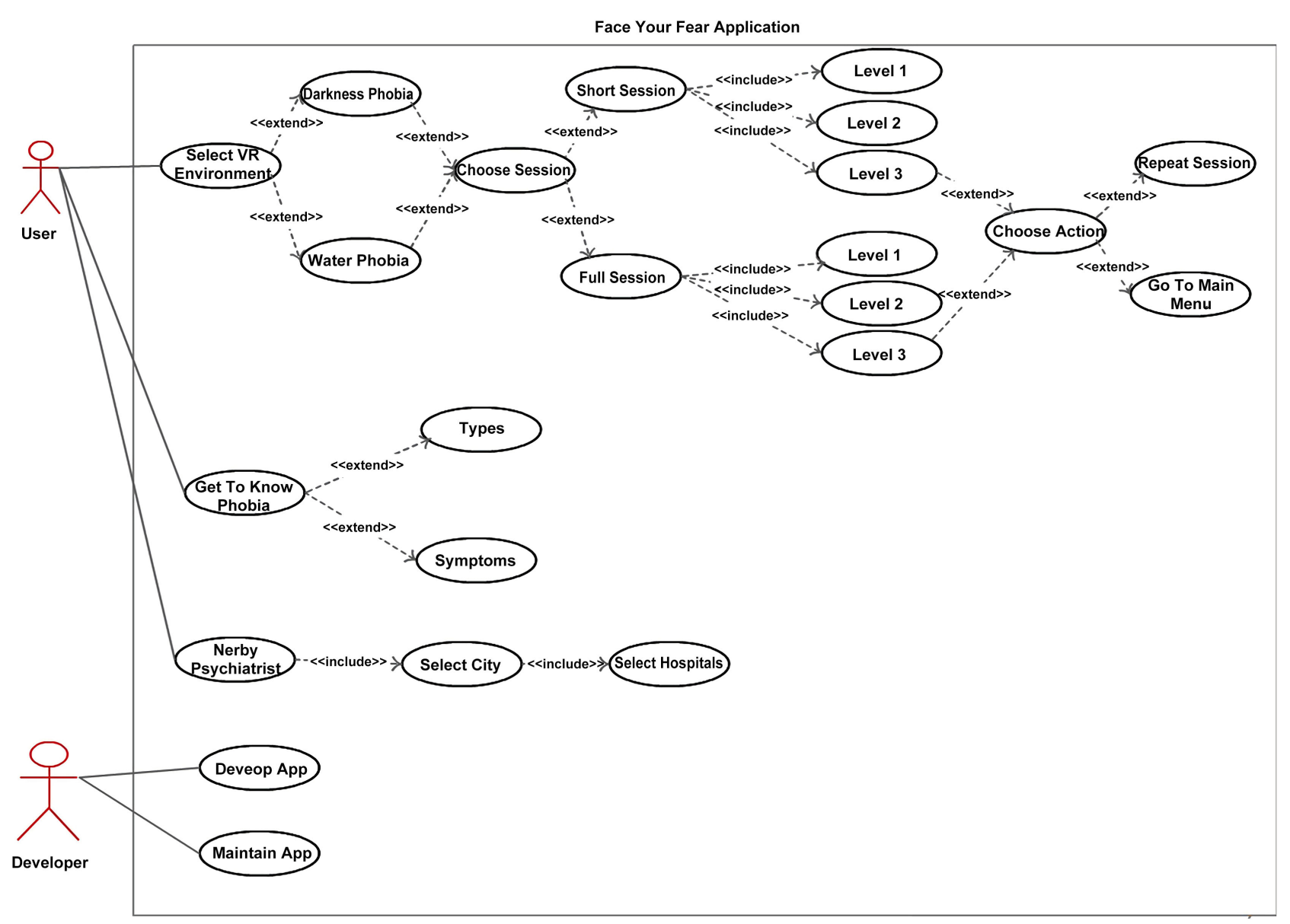

Figure 1. UML (Unified Modelling Language) uses cases diagram.

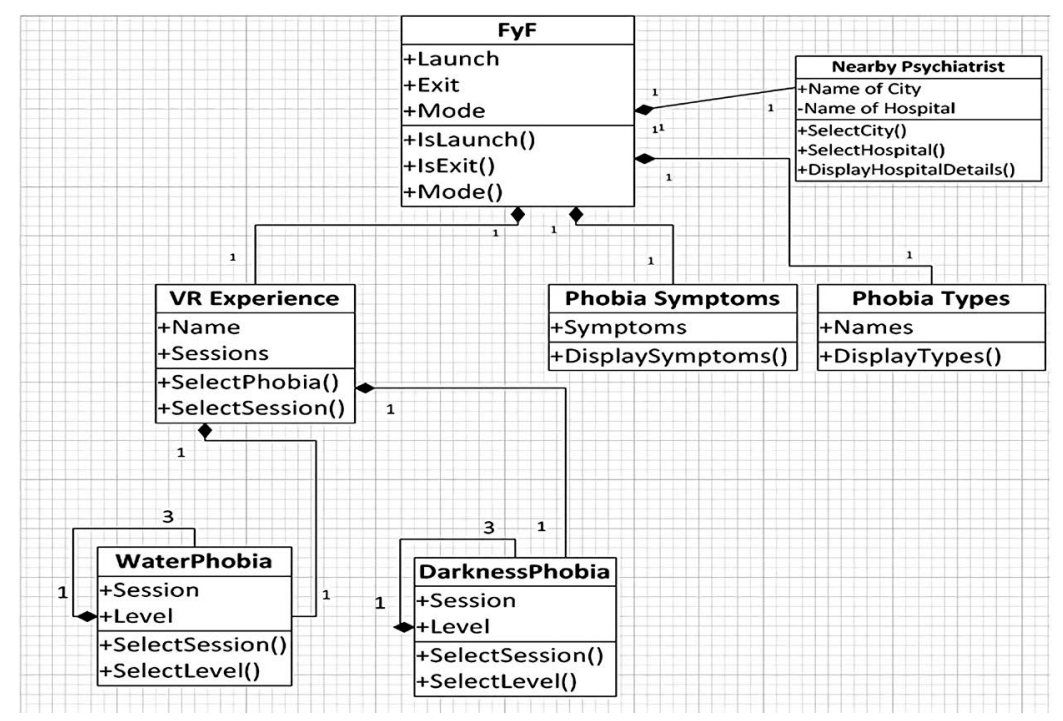

Figure 2. UML classes diagram.

Once the user launches the application, a menu of three options is offered:

- "Experience VR": this possibility allows the user to select phobia environment, that is, aquaphobia, termed as "Water" or achluophobia, named as "Darkness". 
Once the phobia environment is selected, the user will be given two further options, a therapy exposure of short/long session to begin the VR experience with three levels.

- Short Sessions are developed to expose patients to the phobic environment in a suitable duration of time. Per Brenda, the recommended session by professional psychiatrist is $30-45$ minutes by repeated exposure ( 6 - 12 sessions) monthly to phobic stimuli. A short session has the duration of 1 minute and it helps the patient to stimulate with the environment. There are three short session levels and each of which holds of a duration of 5, 10 and 15 seconds, respectively.

- Long Sessions: it has the duration of 30 minutes, as recommended by doctors. The short and long version gives the patient the option to experience VR environment per their level of tolerance. Long sessions have three levels and each level has a specific duration of 5, 10 and 15 minutes, respectively. After the completion of each session, the user can either repeat it or go back to the main menu.

- Before the therapy exposure, the patient can setup a relaxing audio. This capability allows the patient to select the preferred music to be played every 3 minutes to relax. Two types of music can be played: a relaxed version to run throughout the experience to reduce anxiety or an actual sound that enhances the reality of the exposure environment. For example, under the water phobia settings, the patient can hear a lower sound of waves in level 1 (reflecting any beach shore). For the second and third levels, the sound of beach waves will increase and becomes closer to a 3D sound. Regarding the darkness phobia, the music reproduces footsteps sound in a jungle, which will give the patient the experience that s/he is walking. Again, the sound level will increase while more elements can be added for each stage.

- "Find Nearby Psychiatrists Hospital/Clinic": it allows the user for the search of hospitals/clinics that have a psychiatry department in a specific area or city. A screen appears with a drop-down menu that holds a list of cities (in our present application, cities of the UAE such as Sharjah, Fujairah, Al Ain, Dubai, Ras Al-Khaimah or Abu Dhabi). When the user selects a hospital/ clinic, the address and contact details are displayed too. Otherwise, "there is no Psychiatrist in the selected city" message will be displayed.

- "Get to Know Phobia": this option lets the user to read about the different categories of phobia and general information such as their causes.

- "Phobia Symptoms": this preference allows the user to get the common signs of phobia. Occasionally, people are unaware that they are suffering from phobia as they use the avoidance technique of fearful object. These symptoms can help them to understand the implication they face when subjected to certain conditions or objects.

\subsection{Interfaces}

A set of interfaces have been designed by considering the usability and user ex- 
perience, where the displaying menus and functions are clear, easy navigation, well-chosen colors, clear images and suitable text font size. Figure 3 (left) presents the screen when the user first launches this app (it stays for 5 seconds) while Figure 3 (right) presents the main screen with three options: "Experience VR", "Get to Know Phobia", and "Nearby Psychiatrist". It was designed as a wheel with graphical icons for each selection.

The "Experience VR" screen (Figure 4) gives the user two options to select: 1) Water phobia (aquaphobia); 2) Darkness phobia (achluophobia). Afterwards, s/he will be directed to the "Session" screen. For any new VR user, it is advisable that s/he chooses a short session first although real treatment requires long ones (30 minutes, according to doctors). Additionally, the session level time is setup automatically. The scenes for the three levels of aquaphobia and achluophobia are revealed in Figures 4-6, respectively.
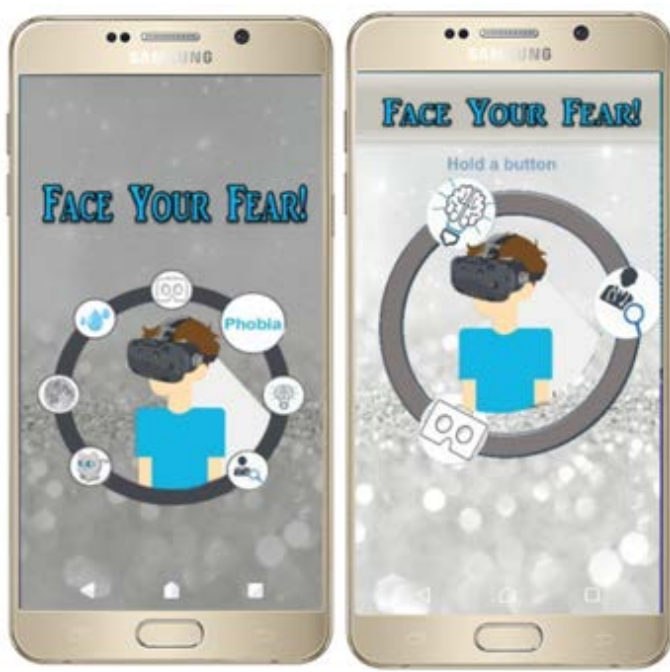

Figure 3. Welcome (left) and main (right) screen.
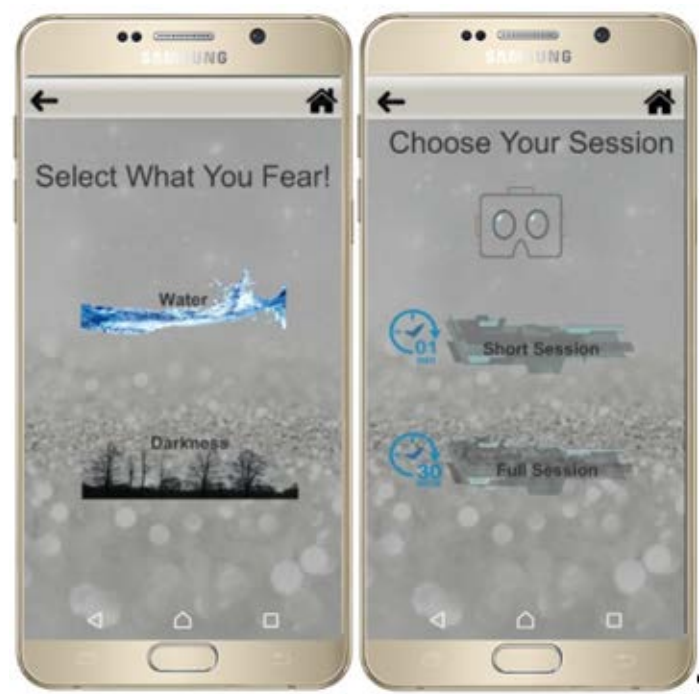

Figure 4. Experience VR" (left) and "Session" (right) screen. 

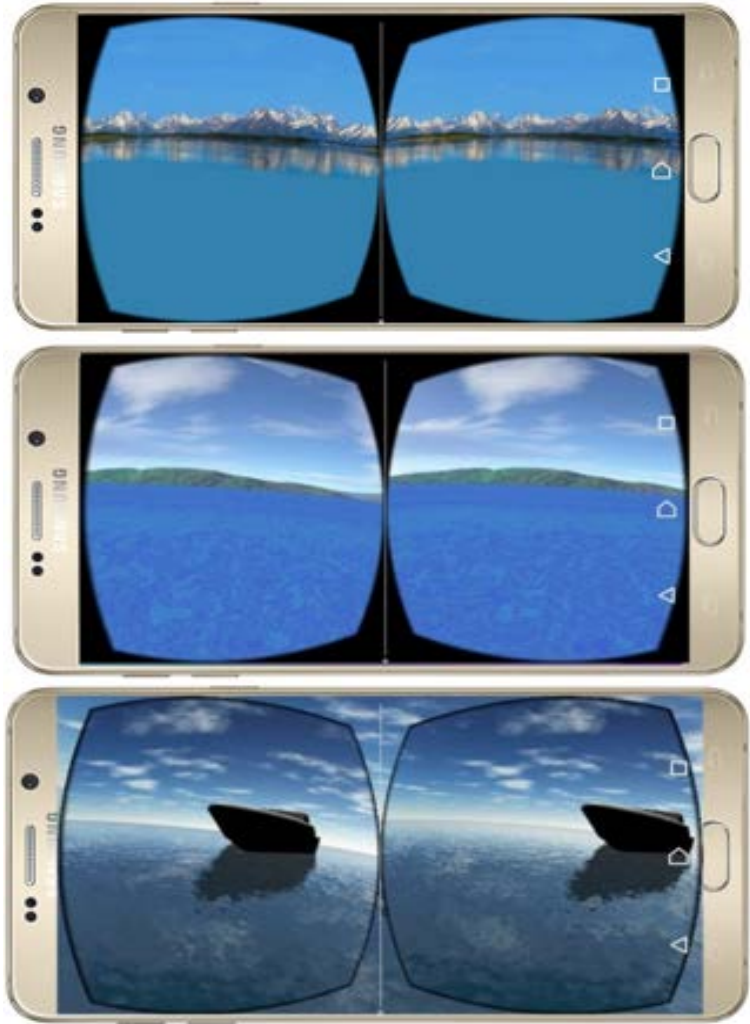

Figure 5. Aquaphobia 1 (top), 2 (middle) and 3 (bottom) levels.

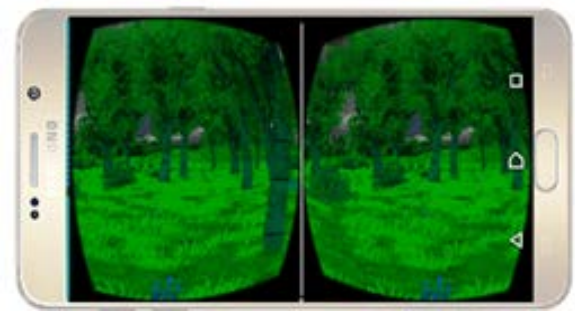

(a)

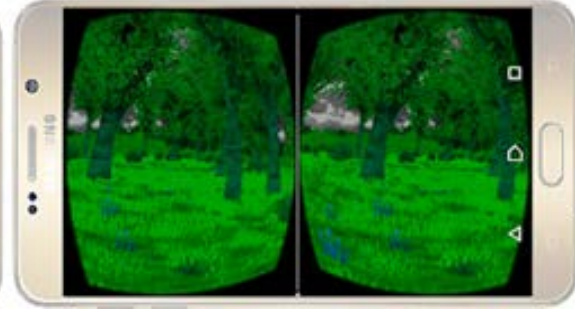

(b)

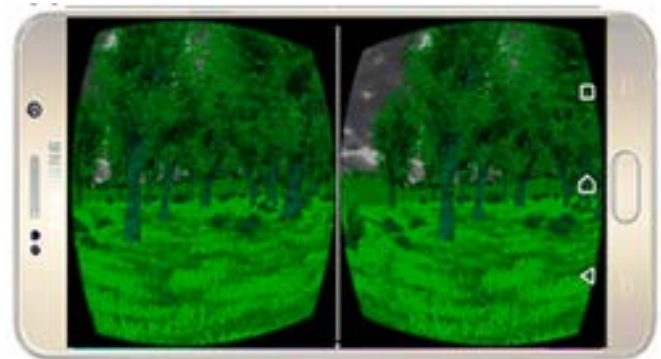

(c)

Figure 6. Achluophobia (a) 1, (b) 2 and (c) 3 levels.

The "Nearby Psychiatrist" helps the user to find the nearest psychiatrics, as shown in Figure 7. For that, the user selects the city from the list box menu. Once the city is selected, another sub-menu regarding all hospitals that holds a psychiatry department is revealed to the user. For usability purposes, we opted 
for list boxes to make it easier rather than typing the name of the city and hospitals and, naturally, to reduce user typos. When a hospital is selected, the address and telephone numbers are disclosed.

The "Get to Know Phobia" screen is shown in Figure 8, where information about common phobia symptoms is presented to the user.

\subsection{Obstacles with Unity and Testing}

FyF was integrated by considering all short and long session levels together including all their interfaces. Yet, two matters should be stressed for future researchers and developers such as the use of different Unity versions. This hurdle cost to the team to redo some environments and interfaces again and again.

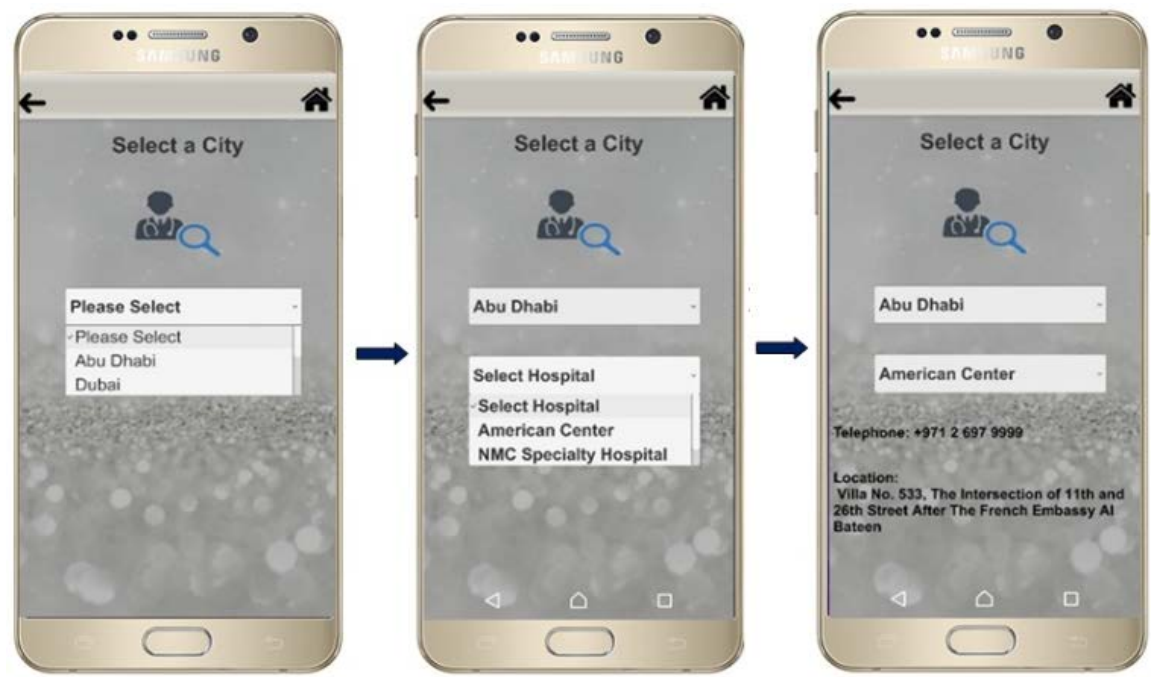

Figure 7. The nearby psychiatrist screens.

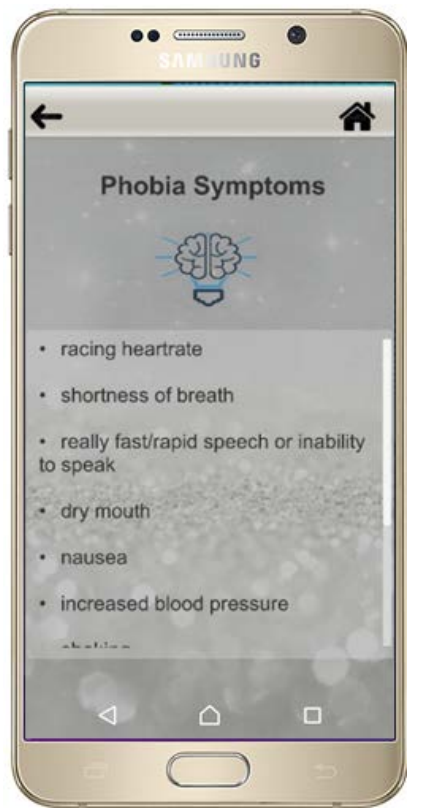

Figure 8. Achluophobia get to know phobia option. 
A second obstacle concerns project ratios, that is, all interfaces were designed on a $1080 \times 1920$ screen resolution but VR environments were designed on Remote Aspect. In Unity, the whole project must respect one ratio only. Otherwise, the interface fitness will be totally wrong.

Testing is an imperative when facing software development since it helps validation and verification of the product. To test FyF, two methods were used: black and white box testing. Black box deals with the verification and validation of inputs and outputs and doesn't require any code testing. Regarding white box, the app code was tested for polymorphism and connection between classes, coherence and inheritance. This means to test timers, loops and movement code, a process that took several days.

\section{Effectiveness Survey}

It is central to include this audience in every aspect to build and produce the right app, including the user experience designer's perspective. Several questions were asked about this mobile software to twenty-three youngsters' users (18 to 23 years old) in a qualitative and quantitative questionnaire while the summary feedback is resumed in the next nine points:

- The interface was quite clean and straightforward with appropriate fonts. Yet, the grey background color should be changed to a blue or a green one since grey represents a dull, dingy, conservative and often associated with loss or depression. On the other hand, blue represents serenity, stability and health while green equals nature.

- The name's app seems good, but the initial logo should be more appealing. Yet, none of the interviewees suggested possible ideas.

- On a 1 - 5 Likert scale, the average experience enjoyment with this system was 3.7 with a standard deviation of 0.6 . Although this sample is quite small, its skewness and kurtosis were close to zero, revealing a Normal distribution of the results.

- The most important ranking features were experience VR and phobia symptoms. Curiously, the nearby psychiatrist was not mentioned at all. A possible reason for this fact encompasses the Islamic culture for this type of service.

- Concerning missing features, acrophobia (fear of heights), arachnophobia (fear of spiders) and claustrophobia (fear of crowded places) should be the following and natural step.

- The navigation ranked 5 on the same 1 - 5 Likert scale since the number of options is still limited.

- On a scale of 1 to 5 , the average rate loading speed of the mobile application was 4.1 with a standard deviation of 0.6.

- In an open-ended question, there was a general discomfort during their experience with the VR glasses used (Google Cardboard) due to the closeness to their eyes. Besides, some of them had medical glasses which did not help ergonomically.

- Generally, these interviewees think that this VR solution may help on the so- 
lution of this common health issue but it will not resolve it for good. Medical and medication should not be forgotten as well.

\section{Conclusions}

Phobia treatment is possible, but it demands patience, perseverance, and time. Unfortunately, people tend to ignore its symptoms. A VR-based mobile app is proposed here to assist phobic patients with an easy use interface to undergo as an initial treatment and by providing them appealing features, such as virtual exposure to the phobic situations or objects, textual description on types of phobia, their symptoms and treatments, searching the nearby psychiatrics, audio that helps them to relax and report submissions capabilities to their doctors. The app, developed to run on both Android and iOS platforms, provides patients the virtual environment to get graded to each stimulus exposure to overcome their fear. Patients may experience a 360-degree view of the phobia agent environment along with sounds to provide a realistic scene. At last, users can turn on music when they feel uncomfortable or move to higher concentration levels.

One of the most common side-effects of phobia is rapid heart rate. To enhance our proposed app, we plan to use a smartwatch to be connected where, during any VR session, the patient's heart rate is being monitored. If the heart rate becomes higher, the patient will be given an option to exit or play a calm audio. If the heart rate exceeds a certain rate (100 bpm for adults), the session will be closed automatically and if the heart rate is in acceptable range, the patient will be able to continue to the next level. This will help patients to keep track of their improvement towards the phobic agent. It should be highlighted here that a key reason why this feature was not still added concerns that not all smartphones can measure the heart rate. Still, there are already some cellular available in the market such as Gear S from Samsung, but this would raise the cost of using this app.

Current state of our proposed app includes two types of phobia: aquaphobia and achluophobia. Other phobia environments might be added easily to our FyF VR application to extend the range of phobias that can offer exposure therapy to patients. It is expected that this app may include others IoT devices, where reports about the patient's heart rate are recorded during each VR experience and the level reached by the patient are sent to his/her psychiatrist. A last improvement regards the search for the nearest psychiatrist using patient's GPS live location, showing to the patient the nearest psychiatry department of any hospital.

\section{Conflicts of Interest}

The authors declare no conflicts of interest regarding the publication of this paper.

\section{References}

[1] Membean (2020) Root Word Phobia Membean. bba.ie/sites/default/files/pictures/eur-rxay/root-word-phobia-membean-18b7ee 
[2] Linares, I.M.P., Trzesniak, C., Chagas, M.H.N., Hallak, J.E.C., Nardi, A.E. and Crippa, J.A.S. (2012). Neuroimaging in Specific Phobia Disorder: A Systematic Review of the Literature. Revista Brasileira de Psiquiatria, 34, 101-111. https://doi.org/10.1016/S1516-4446(12)70017-X

[3] Nordqvist, C. (2014) What Is Stress? How to Deal with Stress. Medical News Today, MediLexicon International Ltd., East Sussex. https://www.medicalnewstoday.com/articles/145855

[4] NHS (National Health Service) (n.d.) Symptoms-Phobia. https://www.nhs.uk/conditions/phobias/symptoms/

[5] Mayo Clinic (2020) Cognitive Behavioral Therapy. https://www.mayoclinic.org/tests-procedures/cognitive-behavioral-therapy/about/p ac-20384610

[6] Khan, T., Johnston, K. and Ophoff, J. (2019) The Impact of an Augmented Reality Application on Learning Motivation of Students. Advances in Human-Computer Interaction, 2019, Article ID 7208494, 14 p. https://doi.org/10.1155/2019/7208494

[7] McLellan, H. (2004) Virtual Realities. In: Jonassen, D.H., Ed., Handbook of Research for Educational Communications and Technology, 2nd Edition, Lawrence Erlbaum Associates, Mahwah, 461-497.

[8] Alraizzah, A., Lamya, F. and Fattouh, L. (2017) Environments and System Types of Virtual Reality Technology in STEM: A Survey. International Journal of Advanced Computer Science and Applications, 8, 77-89.

https://dx.doi.org/10.14569/IJACSA.2017.080610

[9] Douglas Bremner, J., Staib, L., Kaloupek, D., Southwick, S., Soufer, R. and Charney, D. (1999) Neural Correlates of Exposure to Traumatic Pictures and Sound in Vietnam Combat Veterans with and without Posttraumatic Stress Disorder: A Positron Emission Tomography Study. Biological Psychiatry, 45, 806-816.

https://doi.org/10.1016/S0006-3223(98)00297-2

[10] Brooks, T., Camurri, A., Canagarajah, N. and Hasselblad, S (2002) Interaction with Shapes and Sounds as a Therapy for Special Needs and Rehabilitation. 4th International Conference on Disability, Virtual Reality \& Associated Technologies, Veszprém, September 18-21 2002, 205-212.

[11] Novotney, A. (2013) Music as Medicine. https://www.apa.org/monitor/2013/11/music.aspx

[12] Wiederhold, B.K. and Bouchard, S. (2014) Advances in Virtual Reality and Anxiety Disorders. Springer, Boston. https://doi.org/10.1007/978-1-4899-8023-6

[13] La Salandra, A., Frajberg, D. and Fraternali, P. (2020) A Virtual Reality Application for Augmented Panoramic Mountain Images. Virtual Reality, 24, 123-141. https://doi.org/10.1007/s10055-019-00385-X

[14] Torres-Ruiz, M., Mata, F., Zagal, R., Guzmán, G., Quintero, R. and Moreno-Ibarra, M. (2020) A Recommender System to Generate Museum Itineraries Applying Augmented Reality and Social-Sensor Mining Techniques. Virtual Reality, 24, 175-189. https://doi.org/10.1007/s10055-018-0366-Z

[15] Rothbaum, B., Hodges, L. and Kooper, R. (1997) Virtual Reality Exposure Therapy. The Journal of Psychotherapy Practice and Research, 6, 219-226.

[16] Rothbaum, B., Hodges, L., Kooper, R., Opdyke, D., Williford, J. and North, M. (1995) Effectiveness of Computer-Generated (Virtual Reality) Graded Exposure in the Treatment of Acrophobia. American Journal of Psychiatry, 152, 626-628.

https://doi.org/10.1176/ajp.152.4.626 
[17] Virtually Better (n.d.). http://www.virtuallybetter.com/clinic/

[18] Winerman, L. (2005, July) A Virtual Cure: Psychologists Are Using Virtual Reality in Combination with Cognitive-Behavioral Therapy as a Tool to Help People Overcome Phobias. Monitor on Psychology, 36, 87.

[19] Senson, A. (2016) Virtual Reality Therapy: Treating the Global Mental Health Crisis.

https://techcrunch.com/2016/01/06/virtual-reality-therapy-treating-the-global-ment al-health-crisis

[20] Hoffman, H. (2004) Virtual Reality Therapy. Scientific American, Inc., New York, 56-65. https://doi.org/10.1038/scientificamerican0804-58

[21] PHOBOS-Anxiety Management VR Platform (n.d.). https://www.indiegogo.com/projects/phobos-anxiety-management-vr-platform\#/

[22] The Virtual Reality Medical Center (n.d.). http://vrphobia.com/

[23] Cuncic, A. (2021) Beat Social Phobia with Andrew Johnson Review. https://www.verywellmind.com/beat-social-phobia-with-andrew-johnson-3024375

[24] Once Byten Ltd. (2019) Phobia Treatments-Paul McKenna. https://play.google.com/store/apps/details?id=com.oncebyten.pmkphobias\&hl=en\& $\mathrm{gl}=\mathrm{US}$

[25] Blackshear, A. (2012) Cure Phobias and Overcome Fear. https://apkpure.com/cure-phobias-and-overcomefear/com.successtrace.curephobias

[26] Thrive Therapeutic Software Ltd. (2016) Arachnophobia Free. http://apps.apple.com/gb/app/arachnophobia-free/id627935349

[27] Thrive (2016). https://thrive.uk.com/phobia-free-index/\#phobia-free-main

[28] Alterego Games (2019) Project Explained: Spider Phobia VR. https://alteregogames.com/2019/01/03/project-explained-spider-phobia-vr/

[29] VR Heights (2019) VR Heights Phobia. https://play.google.com/store/apps/details?id=com.malumapplications.vrheightpho bia\&hl=en\&gl=US

[30] Kariuki, D. (2016) 4 VR Apps That Help You Be a Better Public Speaker. http://www.hypergridbusiness.com/2016/08/5-vr-apps-that-help-you-be-a-better-p ublic-speaker/ 